\title{
Studies of Ionic Current Rectification Using Polyethyleneimines Coated Glass Nanopipettes
}

\author{
Shujuan Liu, ${ }^{\dagger}$ Yitong Dong, $^{\dagger}$ Wenbo Zhao, $^{\dagger}$ Xiang Xie, ${ }^{\dagger}$ Tianrong Ji, ${ }^{\dagger}$ Xiaohong Yin $^{\dagger}$ Yun Liu, $^{\dagger}$ \\ Zhongwei Liang, ${ }^{\ddagger}$ Dmitry Momotenko, ${ }^{\S}$ Dehai Liang, ${ }^{\dagger}$ Hubert H. Girault, ${ }^{\S}$ and Yuanhua Shao ${ }^{*}{ }^{\dagger}$ \\ ${ }^{\dagger}$ Beijing National Laboratory for Molecular Sciences, College of Chemistry and Molecular Engineering, Peking University, Beijing \\ 100871, China \\ ${ }^{\S}$ Laboratoire d'Electrochimie Physique et Analytique, Ecole Polytechnique Fédérale de Lausanne, Station 6, CH-1015 Lausanne, \\ Switzerland \\ ${ }^{\ddagger}$ Beijing Research Institute of Chemical Industry, No. 14 Beisanhuan Donglu, Chaoyang District, Beijing 100013, China
}

Supporting Information

ABSTRACT: The modification of glass nanopipettes with polyethyleneimines (PEIs) has been successfully achieved by a relatively simple method, and the smallest tip opening is around $3 \mathrm{~nm}$. Thus, in a much wider range of glass pipettes with radii from several nanometers to a few micrometers, the ion current rectification (ICR) phenomenon has been observed. The influences of different $\mathrm{KCl}$ concentrations, $\mathrm{pH}$ values, and tip radii on the ICR are investigated in detail. The sizes of PEIs have been determined by dynamic light scattering, and the effect of the sizes of PEIs for the modification, especially for a few nanometer-pipettes in radii, is also discussed. These findings systemically confirm and complement the theoretical model ${ }^{7,18}$ and provide a platform for possible selectively molecular detection and mimic biological ion channels.

$G$ lass pipettes have been employed for many years as a common tool for manipulating different volumes and types of liquids in laboratories all over the world. When the sizes of glass pipettes go down to micro- and nanometers, there are numerous novel applications. For example, they have been widely used to measure the membrane potentials in patch clamping techniques; ${ }^{1}$ to identify the mechanisms of a facilitated ion transfer (FIT) process at a liquid/liquid interface and measure its kinetic parameters; ${ }^{2}$ and to act as a probe for scanning electrochemical microscopy (SECM) and scanning ion conductance microscopy (SICM) for chemical and high spatial resolution imaging., ${ }^{3,4}$

Ionic current rectification (ICR) is a phenomenon observed in many nanochannels and pores and is often inherently related to the selective transport of ions and molecules. ${ }^{5}$ A remarkable example is the family of inward-rectifying $\mathrm{K}^{+}$channels in cardiac and neuronal cells. ${ }^{6}$ In artificial systems, an ICR usually occurs when the diffuse double layer (DDL) thickness is comparable to the diameter of the nanochannel (or nanopores) and is observed as asymmetric current-voltage curves, with the currents recorded for one voltage polarity higher than the current recorded for the same absolute value of voltage but of opposite polarity. ${ }^{7,8}$ A classic example of ICR by an isolated nanofluidic component was reported over a decade ago using a quartz nanopipette electrode. ${ }^{7}$ Since then, ICR has been observed in silicon-based nanochannels, ${ }^{9,10}$ nanopipettes, ${ }^{7}$ and track-etched polymer membranes. ${ }^{11,12}$ The study of ICR in

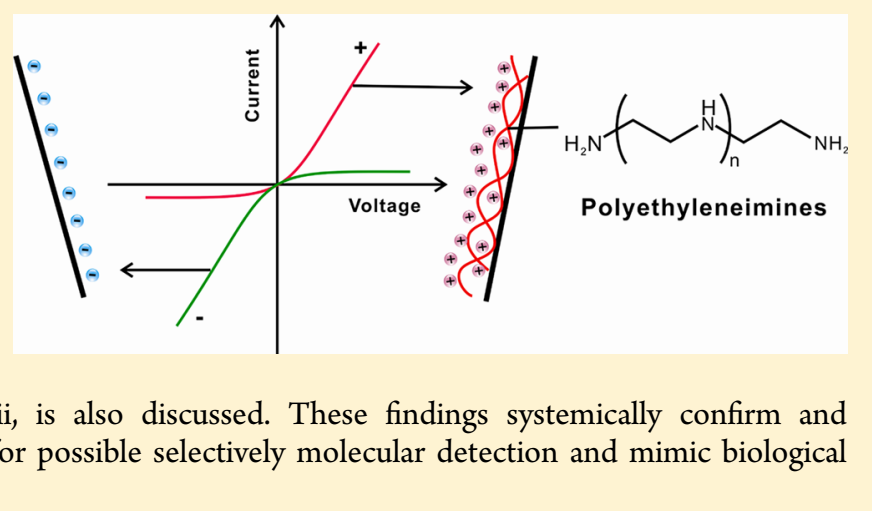

artificial nanochannels (or pores) will help to understand the mechanism of biological ion channels and create new functional nanodevices.

There are several other factors, such as surface charge, pore dimension, shape, etc., that may also influence the extent of ICR. Although the exact mechanism of an ICR is still debatable, several things have been affirmed. First, surface charge can determine the direction of an ICR. Martin et al. ${ }^{12}$ have investigated the role of surface charge on ICR by depositing gold nanotubes into conical polymeric nanopores. ICR could only be observed when $\mathrm{Cl}^{-}$was absorbed, indicating that surface charge was a crucial factor. Ali et al. ${ }^{11}$ have integrated the layer-by-layer polyelectrolyte assemblies into single conical nanopores to study ICR. The charge transport properties of single conical nanopores functionalized with poly(allylamine hydrochloride)(PAH)/poly(styrenesulfonate)(PSS) assemblies are highly dependent upon the number of layers assembled on the pore wall. Second, the size of the pores is also very important. ${ }^{13}$ Generally speaking, an ICR is strongly affected by the relative dimensions of the DDL thickness and pore size and is only observed when the pore size of nanopipette electrodes is comparable to the thickness of the DDL. ${ }^{7}$ By far, most ICR

Received: February 18, 2012

Accepted: June 11, 2012

Published: June 11, 2012 

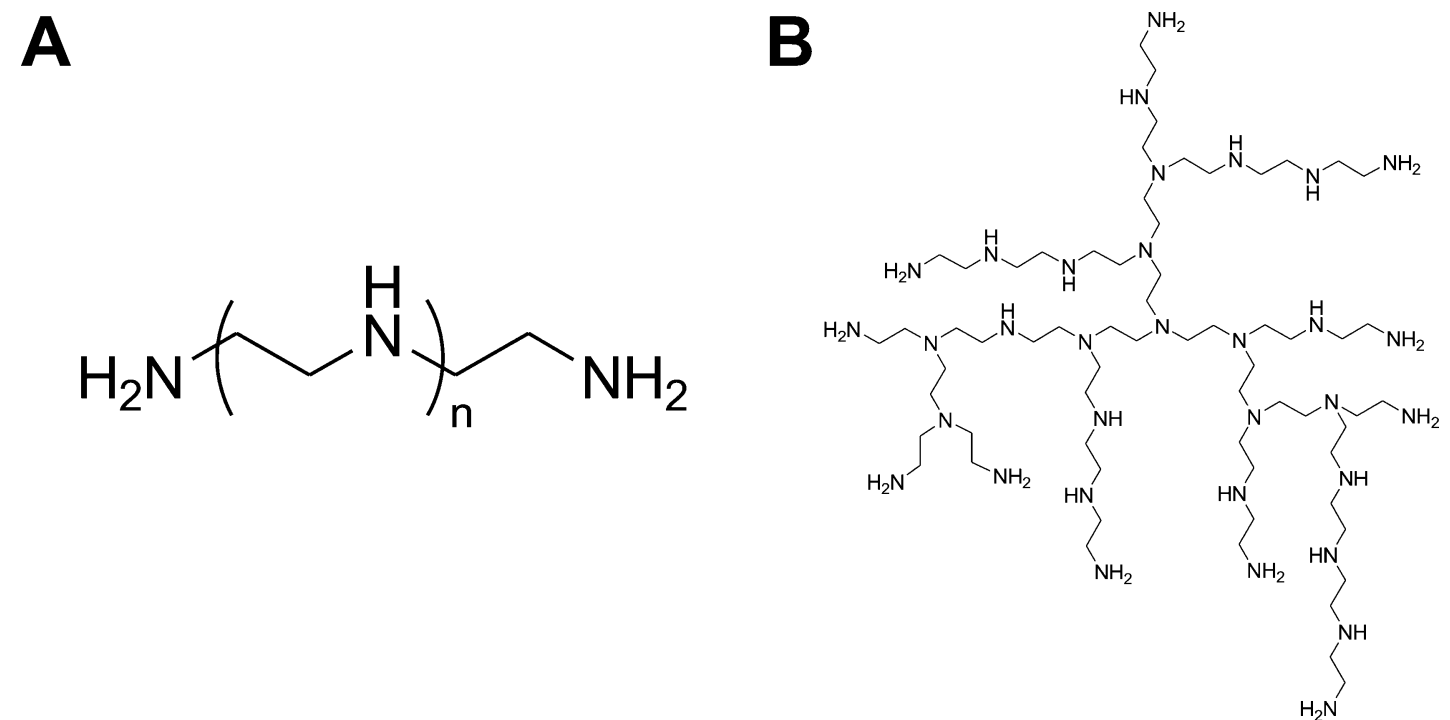

Figure 1. Molecular structures of linear (A) and branched (B) polyethyleneimine (PEIs).

phenomena have been typically observed in nanochannels with an opening diameter of less than $100 \mathrm{~nm}$. The largest pore size reported by now is $2.2 \mu \mathrm{m}$, which was about 500 times larger than the DDL. ${ }^{14}$ Apart from these two factors, the shape of the pore, ${ }^{15}$ scan rates, ${ }^{16,17}$ and other factors ${ }^{18}$ also have significant impacts on the ICR.

Several theoretical models have been proposed to explain the mechanism of ICR. Bard et al. ${ }^{7}$ have considered the asymmetric behavior as the result of a small perm-selective region at the tip coupled with asymmetrical conical regions inside and outside the pore. This region is the link between two conical zones where ionic transport occurs inside and outside the pore. The larger the cross-sectional area taken by the DDL at the tip, the greater the effect is on an ion transport through the orifice. Recent simulations by Girault et al. have confirmed this hypothesis, and they also predict the presence of reverted ICR when using electrolyte solutions at very low ionic strengths. ${ }^{18}$ Siwy and co-workers ${ }^{12}$ have proposed a ratchet model to explain ICR. According to their model, the current is lowered due to an ion trap formed by the applied potential. Woermann ${ }^{19,20}$ has considered the origin of the ICR as three regions at the tip and high and low-conductance states. The pore can be divided into three regions including the bulk region at the base of the cone, a transition zone, and the tip at the small opening of the pore. When a voltage is applied, the transition zone changes its electrical transference number, which leads to a change of conductance of the pore, and consequently the current values are rectified. Quantitative description of the ICR has also been developed by Cervera et $\mathrm{al}^{21}$ and White et al., ${ }^{22}$ and the later model requires just one parameter and provides a clear physical definition.

There have lately been several reports that the ICR can be used for chemical and biosensing. The detection signal is based on current modulations induced by the binding of the analyte to the pore walls which have been modified with certain recognition agents. ${ }^{23-25}$ ICR can also be applied to construct nanodevices that can control ionic flows electrically. Siwy et al. $^{26}$ have fabricated nanopores to build a bipolar ionic transistor that functions in a similar way to its semiconductor bipolar junction counterpart. The gating of the ion current can be realized by changing the $\mathrm{pH}$ and concentration of the bulk electrolyte.

Since the pioneering work of Bard et al., ${ }^{7}$ glass nanopipettes have been used extensively to the study of ICR phenomenon. Chemical modified nanopipettes have been explored to develop chemically or biochemically responsive nanopores. In general, there are two ways to modify the tip surfaces (inner and/or outer walls) of nanopipettes: chemical bonding and electrostatic adsorption. The most common used chemical bonding is the formation of $\mathrm{Si}-\mathrm{O}$ bond on the surface using different silanization reagents. However, it is difficult to modify nanopipette with radius less than $10 \mathrm{~nm}$ in this way. In comparison, the electrostatic adsorption method is easier. Several kinds of polymers, such as poly-L-lysine, ${ }^{9}$ poly(acrylic acid), ${ }^{27}$ and chitosan ${ }^{28}$ et al., have been employed. The smallest diameter of modified nanopipettes reported by these references is about $20 \mathrm{~nm}$.

Polyethyleneimines (PEIs), positively charged polyelectrolytes, are typical water-soluble polyamines with a large number of amine groups on the molecular chain. They behave as effective bases in the aqueous solution and can be protonated at $\mathrm{pH}$ lower than $10^{29}$ As a class of functional water-soluble macromolecules, PEIs have been used in many fields, such as purification and immobilization of biological macromolecules, adsorption and separation of metal ions, and construction of biosensors and drug release. ${ }^{30,31}$ PEIs possess many nitrogen atoms, which make them good chelating agents for metal ions, and can be further modified. Moreover, PEIs can interact with negatively charged DNA through electrostatic interaction to form nanocomplexes, and they have been widely attempted to use as a gene delivery system. ${ }^{32}$ Choosing of the PEIs as modifiers here may help to establish basic understandings of the mechanism of ICR and extend the application of the PEIs and nanopipettes.

Herein, we develop a simple and high success rate method of modification of glass nanopipettes with polyethyleneimine (PEIs) and observe ICR phenomena in a much wider range of radii of glass pipettes (from several nano- to few micrometers) and much wider concentration range of $\mathrm{KCl}$. The success rate of modification of nanopipettes is about $50 \%$, and the smallest pipet can be modified with PEIs is around $3 \mathrm{~nm}$. In some rather 


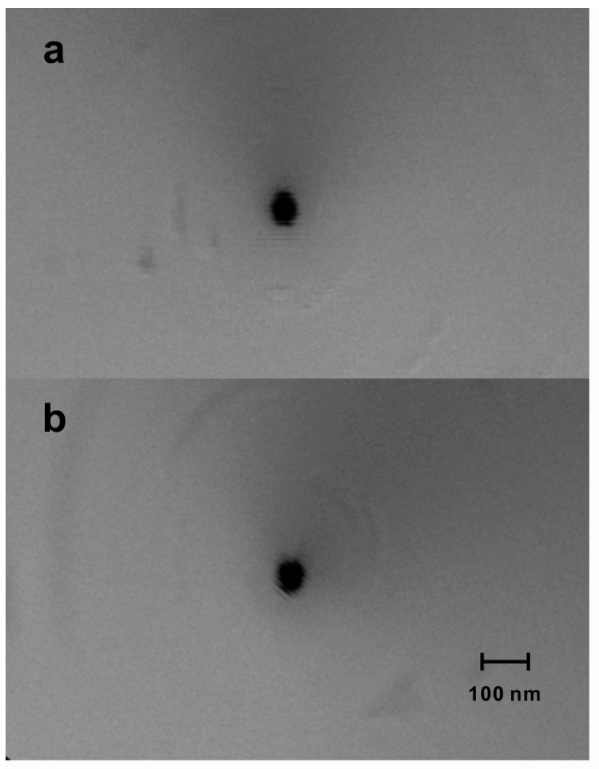

A a

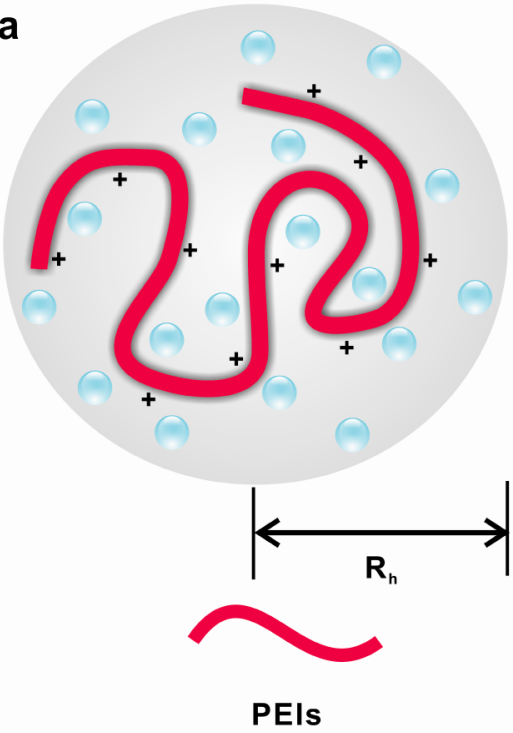

b

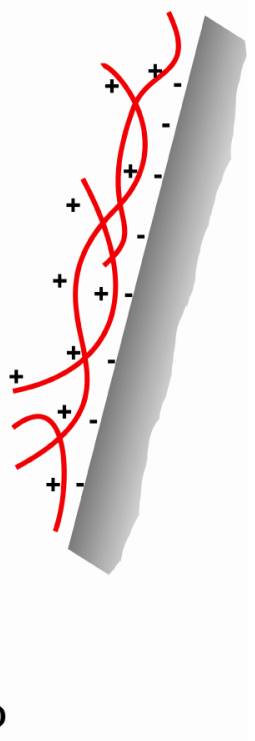

B

Figure 2. (A) The SEM images of a nanopipette before (a) and after (b) PEIs coated. The tip radius is about $30 \mathrm{~nm}$. (B) Schematic representation of the hydrated form of PEIs in free solution (a) and the condensed form of PEIs on the glass surface after modification (b).

low concentration cases, the DDL thickness is larger than the tip radius. From the influences of $\mathrm{pH}$ on the ICR, the values of $\mathrm{p} K_{\mathrm{a}}$ of PEIs on the surface are estimated to be 8.2 and 3.5, respectively.

\section{EXPERIMENTAL SECTION}

Chemicals. Potassium chloride $(\mathrm{KCl}, \geq 99.5 \%$, Beijing Chemical Co.), dibenzo-18-crown-6 (DB18C6, $\geq 98 \%$, Alfa Aesar), polyethyleneimine (PEI, linear, M. W. 25000, Alfa Aesar \& branched, M. W. 25000, Aldrich), potassium tetrakis(4-chlorophenyl)-borate (KTPBCl, $\geq 98 \%$, Aldrich), and bis(triphenylphosphoranylidene)-ammonium chloride (BTPPACl, $\geq 98 \%$, Aldrich) were used as received without further purification. 1,2-Dichloroethane (DCE, $\geq 99 \%$, Beijing Chemical Co.) was washed with triply distilled water before use. Bis-(triphenylphosphoranylidene)-ammonium tetrakis(4chlorophenyl) borate (BTPPATPBCl) was synthesized by metathesis of equimolar solutions of BTPPACl and $\mathrm{KTPBCl}^{2,33}$ The salts were recrystallized from acetone and then dried in an oven at $95{ }^{\circ} \mathrm{C}$ for $24 \mathrm{~h}$. All aqueous solutions were prepared from triply distilled water.

Fabrication and Modification of Glass Pipettes. Different size pipettes were fabricated by a $\mathrm{CO}_{2}$-laser-based pipet puller (P-2000, Sutter Instrument Co.) with quartz capillaries $(0.7 \mathrm{~mm} \mathrm{I.D.} \mathrm{and} 1.0 \mathrm{~mm}$ O.D.) from the same company of the puller. The program used in the fabrication of glass pipettes of several nanometers in radii is shown in Program I

$$
\begin{aligned}
(\text { Cycle } 1) \text { heat } & =800, \text { filament }=5, \text { velocity }=20, \text { delay } \\
=128, \text { pull } & =50 ; \\
(\text { Cycle } 2) \text { heat } & =850, \text { filament }=4, \text { velocity }=15, \text { delay } \\
=130, \text { pull } & =175
\end{aligned}
$$

(Program I)
The aqueous solution was backfilled into the pipettes using a microfill needle, and then the pipettes were tapped to drive out the air bubbles and checked under a microscope (BX-51, Olympus). The $\mathrm{pH}$ value was determined by a $\mathrm{pH}$ meter (PHSJ-3F, Shanghai Precision and Scientific Instrument).

Two types of PEIs with different structures (Figure 1), linear and branched, of the same molecular weight (M. W. 25000) were employed. The modification procedure was as follows. First, $0.6 \mu \mathrm{L}$ of $0.1 \%$ PEIs aqueous solution was backfilled into the tip of pipet, and then the pipettes were placed in air for 30 min to let PEIs interact with the inner walls. Finally the pipettes were baked at $120{ }^{\circ} \mathrm{C}$ for $2 \mathrm{~h}$ to remove water. The inject procedure should be carried out quickly, and the amount of PEIs solution injected should be the same among the pipettes to make sure the modification results were repeatable. The waiting time of $30 \mathrm{~min}$ after injection was also very important. During this period of time, the PEIs can interact with the inner wall thoroughly, and a higher success rate can be achieved. Considering both the efficiency and the success rate, we waited $30 \mathrm{~min}$ to put the nanopipettes in the oven after the injection of PEIs solution in the experiment.

The two structures of PEIs have different physical properties. Linear structured PEI takes the form of a white powder which can be easily dissolved in hot water. Branched PEI is a sticky liquid and may block the opening of the pipettes now and then. Hence, we employed linear structured PEI to accomplish most of the experiments and used the branched one just as a comparison.

Figure 2A demonstrates a comparison of the pipet tip before and after the modification. The scanning electron microscopic (SEM) images (obtained by using XL-30 ESEM-FEG, FEI) show that the coating process has no influence on the diameter of the pipet (or the layers are too thin to be observed by the SEM under the conditions), which means the current response changes are caused mainly by the coating on the inner wall of 

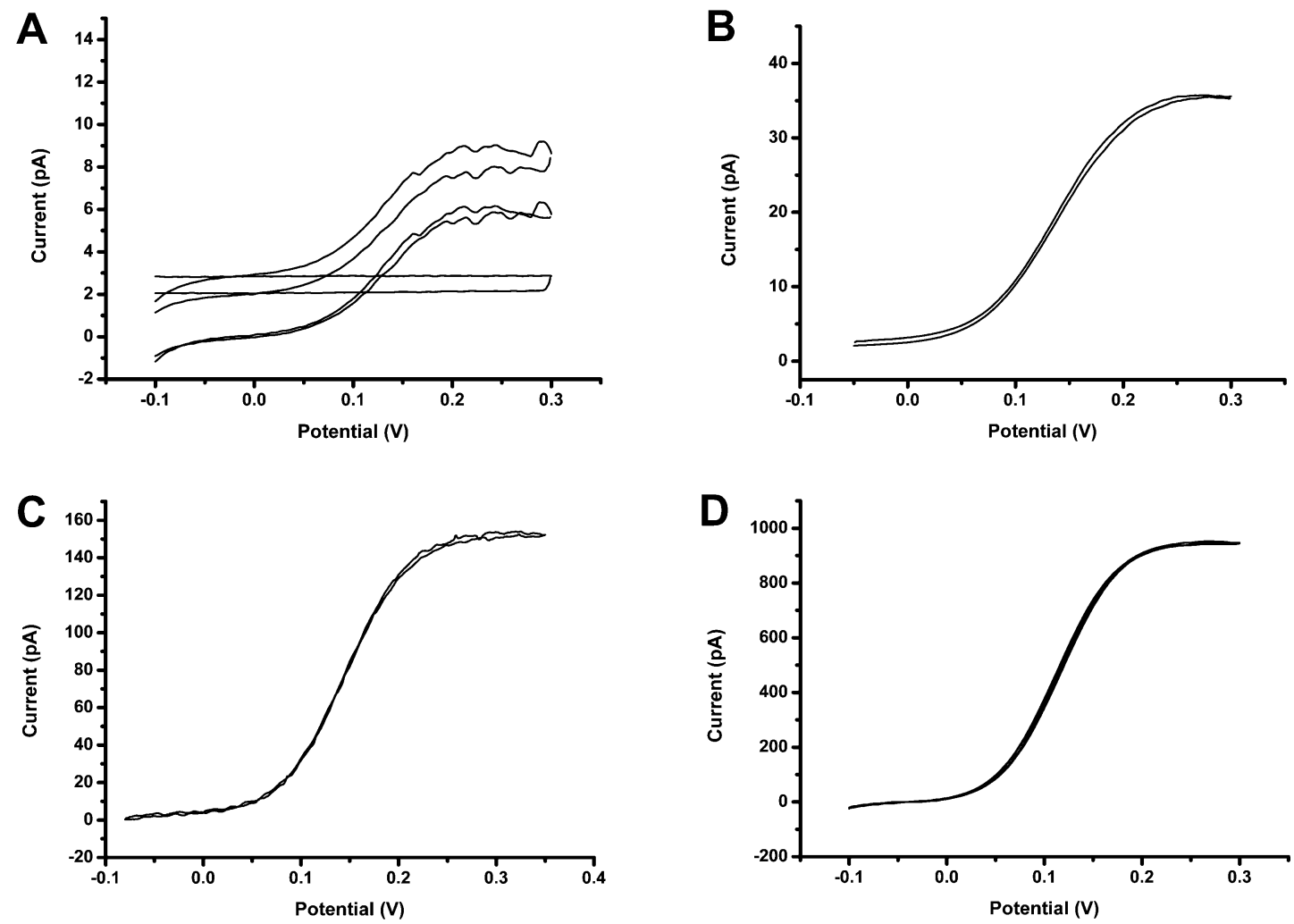

Figure 3. The cyclic voltammograms of $\mathrm{K}^{+}$transfer from the aqueous phase to the DCE phase facilitated by DB18C6 using Cell 1 . The sweep rate was $50 \mathrm{mV} / \mathrm{s}$. (A) $r=3 \pm 1 \mathrm{~nm}(n=3)$. (B) $r=22 \pm 6 \mathrm{~nm}(n=8)$. (C) $r=110 \pm 16 \mathrm{~nm}(n=7)$. (D) $r=744 \pm 29 \mathrm{~nm}(n=3)$.

the pipettes. Their hydrodynamic radii $R_{\mathrm{h}}$ have been evaluated using dynamic light scattering (DLS) by a commercialized spectrometer (Brookhaven Inc., Holtsville, NY) with a $100 \mathrm{~mW}$ solid-state laser (GXC-III, GNI, Changchun) operating at 532 $\mathrm{nm}$ which was used as the light source and are 4.1 and $5.3 \mathrm{~nm}$, respectively (For more details see the Supporting Information, SI.). Figure $2 \mathrm{~B}$ shows the schematic representation of the $R_{\mathrm{h}}$ and the possible way of coating PEIs on the inner wall of the pipet.

Current-voltage curves were obtained with an electrochemical workstation (BAS 100B, Bioanalytical Systems). An $\mathrm{Ag} / \mathrm{AgCl}$ electrode was inserted inside of the pipet, and the other $\mathrm{Ag} / \mathrm{AgCl}$ reference electrode was placed in the external electrolyte bath. The solution inside the pipet was the same as the bath solution. Due to fluctuations in the tip fabrication and modification, the experimental data may vary from each other a little, and the results have been repeated at least 3 times using the same size of pipettes.

\section{RESULTS AND DISCUSSION}

Characterization of Glass Nanopipettes. Using the P2000 puller, we can fabricate nanopipettes with radii of several $\mathrm{nm}$ using a proper program. For one pulling, two almost identical nanopipettes can be made. One can be used for the electrochemical characterization of its radius, and another is for further modification. It is usually not easy to determine the size of nanopipettes by SEM when their radii are less than $20 \mathrm{~nm}$ because they are nonconductive. The radii of the glass nanopipettes (noncoated) are evaluated here by steady-state voltammetry of the facilitated $\mathrm{K}^{+}$transfer at the water/1,2dichloroethane (W/DCE) interface by DB18C6. This methodology has been proved to be an effective way to determine the radius of a nanopipette. ${ }^{34-37}$ The electrochemical system for characterization of nanopipettes can be represented as Cell 1

$$
\begin{aligned}
& \mathrm{Ag} / \mathrm{AgTPBCl} / 2 \mathrm{mM} \mathrm{DB} 18 \mathrm{C} 6+2 \\
& \mathrm{mM} \mathrm{BTPPATPBCl}(\mathrm{DCE}) / / 100 \mathrm{mM} \mathrm{KCl}(\mathrm{W}) / \mathrm{AgCl} / \mathrm{Ag}
\end{aligned}
$$

Here $\mathrm{TPBCl}$ refers to tetrakis(4-chlorophenyl)borate, and BTPPATPBCl stands for bis(triphenylphosphoranylidene)ammonium tetrakis(4-chlorophenyl)borate, a lipophilic salt used to provide ionic conductivity to the DCE phase.

Because the concentration of $\mathrm{K}^{+}$inside the pipet is much higher than that of DB18C6 in the DCE, the steady-state current is limited by the diffusion of DB18C6 to the tip. The empirical equation proposed by Girault et al. ${ }^{34}$ can be used to characterize the effective radius of a pipet

$$
I_{\mathrm{ss}}=3.35 \pi n \mathrm{FD} \mathrm{cr}
$$

Here $I_{S S}$ is the steady-state current, $n$ is the charge of the transferred ion, $F$ is the Faraday constant, $D$ is the diffusion coefficient, $c$ is the bulk concentration of DB18C6 in the DCE phase, and $r$ is the inner pipet radius.

Figure 3 shows the cyclic voltammograms obtained for the $\mathrm{K}^{+}$transfer facilitated by DB18C6 at the W/DCE interface using the nanopipette voltammetry with different sizes. Due to the inherent charging current, a background subtraction has been used to obtain high quality voltammograms for the several nanometer-sized pipettes. Because the tip cannot be exactly the same from each other even under the same pulling conditions, $^{38}$ the radius is averaged from at least three measurements using the glass pipettes supposed to be the same radii. The effective radii of the nanopipettes can be calculated from Figure 3 as $3 \pm 1 \mathrm{~nm}(n=3), 22 \pm 6 \mathrm{~nm}(n=$ 



Figure 4. Current-voltage curves of noncoated and PEIs-coated nanopipettes in $1 \mathrm{mM} \mathrm{KCl}(\mathrm{pH}=6.5)$. Scan rate, $50 \mathrm{mV} / \mathrm{s}$. (A) $r \approx 700 \mathrm{~nm}$. (B) $r$ $\approx 100 \mathrm{~nm}$. (C) $r \approx 20 \mathrm{~nm}$. (D) $r \approx 4 \mathrm{~nm}$. The inset is the magnified view of curve 3 .

8), $110 \pm 16 \mathrm{~nm}(n=7)$, and $744 \pm 29 \mathrm{~nm}(n=3)$, respectively.

The ICR Behaviors Obtained with and without Modification of the Glass Pipettes. Figure 4 shows the current responses change upon the application of electric potential before and after modification of the glass pipettes with the linear PEIs. For various types of $\mathrm{SiO}_{2}$ materials, the isoelectric point is normally within the $\mathrm{pH}$ range of 1 to $4 .{ }^{39}$ The $\mathrm{pH}$ value of the $1 \mathrm{mM} \mathrm{KCl}$ solution is around 6.5. The surface silanol groups of the pipettes dissociate primarily, ${ }^{40}$ which make the surface negatively charged. In Figures $4 \mathrm{~A}$ and $4 \mathrm{~B}$, the $i-\mathrm{V}$ relationships of noncoated pipettes are more or less linear. This is because the radii of the pipettes are relatively large in a range of several hundred nanometers. According to the previous report, ${ }^{7}$ ICR can usually be observed with radii of dozens of nanometers for unmodified glass pipettes under these experimental conditions. It can be seen from Figure 4C that the $i-\mathrm{V}$ curves bend downward for the bare glass nanopipette, which is in good agreement with the results observed by Bard et al. $^{7}$ due to the effect of the DDL. After glass surface modification the $i-\mathrm{V}$ curve becomes nonlinear, which means the current is rectified. The current is significantly increased at the opposite side (see the dotted line in Figure 4C). The $i-\mathrm{V}$ curve (shown as dotted line) in Figure 4C is just the opposite to the $i$ $\mathrm{R}$ relationship of ICR of unmodified glass nanopipettes (solid line) and rectification effect is stronger. Therefore, the direction of ICR indicates that the PEIs have been coated onto the surface. This is because after the modification the surface charges are changed from negative to positive, and the charge density is also increased. The change of the direction of ICR can be explained using the permselectivity approach. ${ }^{7,18}$ After modification with PEIs, the inner wall is positively charged. Consequently, there shall be a permselective region where $\mathrm{Cl}^{-}$ is accumulated and $\mathrm{K}^{+}$is depleted. When a positive potential is applied, $\mathrm{Cl}^{-}$has to move into the tip to keep electroneutrality. Because of the asymmetric shape of nanopipettes, the migration of $\mathrm{Cl}^{-}$from a hemispherical field outside the tip is less hindered and produced a large current.

This modification method has also been applied to nanopipettes with radii around $3 \mathrm{~nm}$. The modification of nanopipettes has always been very difficult due to its extremely small diameters and special geometry. ${ }^{35,41,42}$ Using this method we have successfully modified the inner surface of nanopipettes. From Figure $4 \mathrm{D}$ it can be seen that after modification, the current is rectified greatly. One thing to be noticed is that the current after modification reduced a lot compared to bare pipet. This is probably because the modified layers of PEIs blocking part (or fully) of the tip of pipet since its hydrodynamic radius $R_{\mathrm{h}}$ is about $4.1 \mathrm{~nm}$ (see the $\mathrm{SI}$ ). We have also tested the influence of the concentration of PEIs solution. When PEIs concentration is $0.1 \%$, the $i-\mathrm{V}$ curve remained essentially linear, and a very clear ICR can be observed after modification with much higher (e.g., 0.9\%) concentration of PEIs. Using PEIs solution with higher concentrations, more PEIs molecules can be immobilized onto the surface and the density of positive charges would also be larger. Higher PEIs concentration may make the modification more effective, but it will increase the chance of blocking the tip at the same time. In addition, the size 

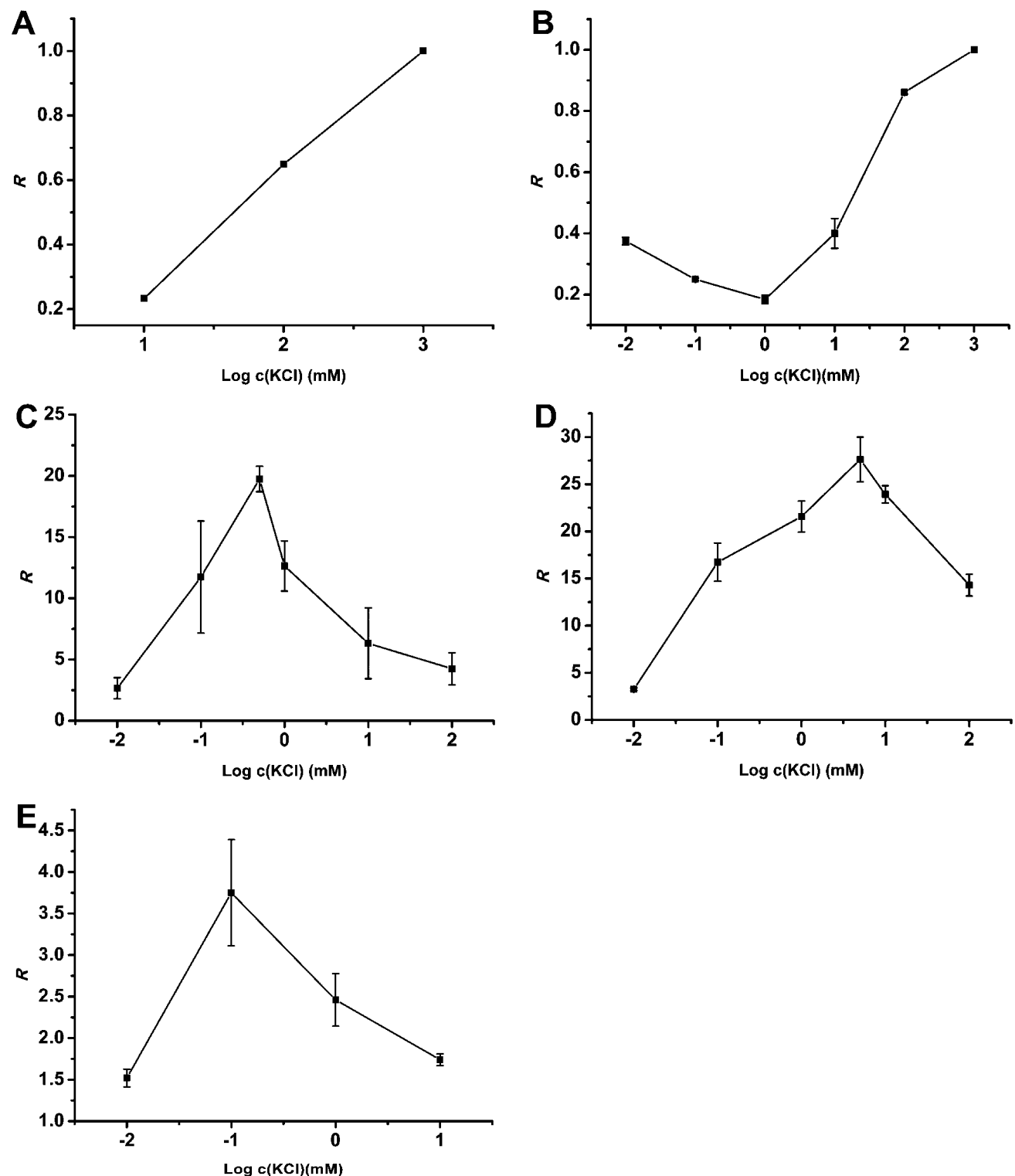

Figure 5. Dependence of ion current rectification on $\mathrm{KCl}$ concentration for bare and PEIs-coated nanopipettes. $R=i_{+} / i_{-} . \mathrm{KCl}$ concentration varies from $0.01 \mathrm{mM}$ to $100 \mathrm{mM}$. Scan rate is $50 \mathrm{mV} / \mathrm{s}$. (A) Data from the work of Bard and co-workers. ${ }^{7} R$ is calculated by the current values at $\pm 0.5 \mathrm{~V}, r$ $\approx 20 \mathrm{~nm}$. (B) Bare nanopipettes, $r \approx 20 \mathrm{~nm}$. (C) PEIs-coated nanopipettes, $r \approx 1 \mu \mathrm{m}$. (D) PEIs-coated nanopipettes, $r \approx 100 \mathrm{~nm}$. (E) PEIs-coated nanopipettes, $r \approx 4 \mathrm{~nm}$.

reported here is the radius of hydrated form of PEIs in free solutions. When PEIs were immobilized on the glass surface in a limited space, they would probably exist in a more condensed form, which means the size should be even smaller (see Figure 2B). The successful modification ratio for nanopipettes with radii of several nanometers is good, and about half of the pipettes can be modified successfully by this method.

The Current Response Dependence on $\mathrm{KCl}$ Concentration. Here, we define a rectification factor $(R)$ as the ratio of positive current, $i_{+}$, to negative current, $i_{-}$, at the same absolute voltage amplitudes to quantify the extent of rectification. In this work we choose the current values at $\pm 1 \mathrm{~V}$ and $\pm 0.5 \mathrm{~V}$ (for branched PEIs coated nanopipettes) to calculate $R$. In our experiments, $R$ should be bigger than 1 if the inner wall of the pipet was positively charged, and larger $R$ values indicate a stronger rectification.
Figure 5 shows the relationship between $R$ and $\mathrm{KCl}$ concentration. The concentration of $\mathrm{KCl}$ solution varied from $0.01 \mathrm{mM}$ to $100 \mathrm{mM}$. The change of $R$ should be related to the thickness of DDL. The thickness of DDL $(\delta)$ at a planar surface for a $1: 1$ electrolyte is given by ${ }^{43}$

$$
\delta=1 / \kappa=\left(2 c^{o} z^{2} e^{2} / \varepsilon \varepsilon_{0} k T\right)^{-1 / 2}
$$

where $c^{\circ}$ represents the concentration of species of charge $z$ in a phase of dielectric constant $\varepsilon$ at temperature $T$, and $e, \varepsilon_{0}$, and $k$ are the electronic charge, permittivity of free space, and the Boltzmann constant, respectively. At $25{ }^{\circ} \mathrm{C}$, for aqueous solutions it can be written as

$$
1 / \kappa=3.1 \times 10^{-8} / c^{1 / 2}
$$

where $c^{*}$ is the concentration (in $\mathrm{mol} / \mathrm{L}$ ). 
Figure $5 \mathrm{~A}$ is the relationship of $R$ and concentration reconstructed based on the data from the work of Bard and co-workers. ${ }^{7}$ They have tested three different concentrations of $\mathrm{KCl}$, which were $0.01,0.1$, and $1 \mathrm{M}$, respectively. Here, we use current values at $\pm 0.5 \mathrm{~V}$ to calculate $R$. Because the pipettes were not modified at near neutral $\mathrm{pH}$ solution, they should have negative charges on the wall. Therefore, the $i$-V curve bend downward and $R$ is smaller than 1 . Smaller $R$ means stronger ICR here because the bare pipet has negative charges. According to their results, the ICR became stronger along with the decrease of the $\mathrm{KCl}$ concentration. They thought this was because the more cross-sectional area taken by the DDL at the tip, the greater the effect on ion transport through the opening. The tip radius was about $20 \mathrm{~nm}$, so that the cross-sectional areas at the orifice were 28,10 , and $3 \%$ for the corresponding electrolyte concentrations. The strongest ICR phenomenon was observed when the concentration was $0.01 \mathrm{M}$. However, in all the cases they investigated, the DDL was less than the tip radius. We repeat their experiment with wider concentration ranges. It is clear from Figure 5B that a more or less linear line can also be observed from 0.001 to $1 \mathrm{M}$ of $\mathrm{KCl}$. If the $\mathrm{KCl}$ concentration is further decreased, a peak shape relationship can be observed. When DDL is less than the radius for the case of bare nanopipettes, lower concentration corresponds to stronger rectification. When DDL is larger than the radius, $R$ increases along with decreasing $\mathrm{KCl}$ concentration. ${ }^{18}$

Figures 5C and 5D are obtained using PEIs modified nanopipettes with radii of $1 \mu \mathrm{m}$ and $100 \mathrm{~nm}$. The observation of maximum $R$ agrees with the results reported by previous work $^{13,18,22}$ and can be explained by conductivity changes at the tip because of the increase of DDL size. According to eq 1 , the thickness of DDL is calculated as $98,31,14,10,4.4,3$, and 1 $\mathrm{nm}$ in $0.01,0.1,0.5,1,5,10$, and $100 \mathrm{mM} \mathrm{KCl}$ solutions, respectively. In Figure 5C, $R$ reaches a peak value when the concentration is $0.5 \mathrm{mM}$. If we assume that when the concentration is about $0.5 \mathrm{mM}$, the most cross-sectional area is taken by the DDL. Here we define an effective radius as the characterized radius of the modified nanopipettes, which is the overall representation of radius, surface charge, and tip shape, and it can be approximately estimated that the effective radius of the tip is $14 \mathrm{~nm}$. Compared with the original orifice, which is nearly $1 \mu \mathrm{m}$, the effective radius is reduced by approximately 2 orders of magnitude.

When nanopipettes with radii of $100 \mathrm{~nm}$ are used, the peak value moves toward higher concentration (see Figure 5D). This is because a smaller radius needs smaller DDL to reach the strongest ICR. From the above discussion, it can be forecasted that the peak should be reached around several nanometers. The results in Figure 5C agree well with the conclusion we reached before. It can be calculated that the effective radius is about $4.4 \mathrm{~nm}$, and it is also reduced about 2 orders of magnitude.

When we use nanopipettes of several nanometers, the $R$ values become rather small (see Figure $5 \mathrm{E}$ ). $R$ changes from 1.5 to 3.7. The peak moves toward lower concentration, which is contradictory with the discussion above. One possible reason is that the concentration of PEI solution used to modify the nanopipettes is much higher here, and the charge density is also larger. So the effective radius should be reduced by even more times. That is why $R$ reaches the peak value at smaller $\mathrm{KCl}$ concentration compared with other nanopipettes. Another possible reason is that it is likely that one PEI molecule can bridge across a nanopipette tip and that the inner space near a pipet tip eventually is filled with many polymer molecules and their counterions. The mechanism is different from abovementioned one, and perhaps it is similar to a monolithic column for electrophoresis in this case. It could be also an angle effect related to the fabrication of pipettes with small orifice. This matter is still needs to be further carefully explored.

The Current Response Dependence on pH Values. The $\mathrm{KCl}$ solution without $\mathrm{pH}$ adjustment is slightly acidic ( $\mathrm{pH}$ $=6.5$ ) in our experiments. The solution $\mathrm{pH}$ is adjusted by the partial replacement of $\mathrm{KCl}$ with either $\mathrm{HCl}$ or $\mathrm{KOH}$. There is no sudden change during the titration of PEIs, ${ }^{44}$ and this may relate to that the PEIs should have possibly more than one value of $\mathrm{p} K_{\mathrm{a}}$. The $\mathrm{p} K_{\mathrm{a} 1}$ of the PEIs is about 10 in the aqueous solution from a previous titration report. ${ }^{44}$ Under a certain range of acidic and slightly basic environment, the amine groups should be ionized and carry positive charges. When $\mathrm{pH}$ is adjusted to above $8, R$ is smaller than 1 , which means the inner wall becomes negatively charged. Under basic conditions, when $\mathrm{pH}>8$, most amine groups on the surface remains unionized. The dissociation of silanol groups contributes to the negative surface charges under this condition. $R$ begins to rise when $\mathrm{pH}$ is lower than 8 , which means that at this moment most amine groups of PEIs start to be protonated and brings positive charges onto the wall. From Figure 6, we may say that

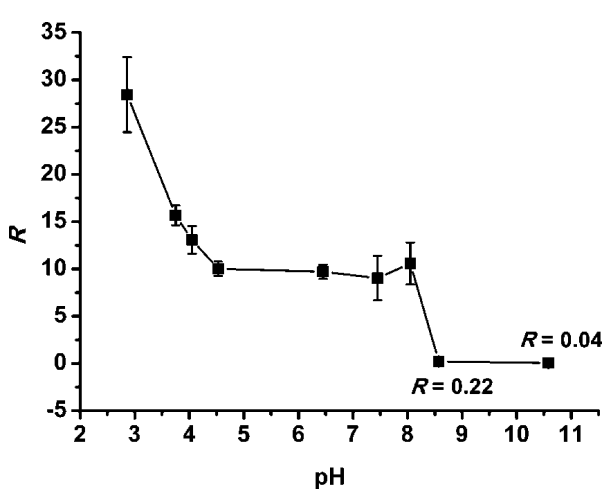

Figure 6. Dependence of ion current rectification on solution $\mathrm{pH}$ for the PEIs-coated nanopipettes $(r \approx 750 \mathrm{~nm}) . R=i_{+} / i_{-}$.

the $\mathrm{p} K_{\mathrm{a} 1}$ of PEIs is about 8.2 when the PEIs are modified onto the surface. The $\mathrm{p} K_{\mathrm{a} 1}$ of PEIs is reduced from 10 to 8.2 after the modification. This is because part of amine groups are attached on to the surface and more acidic environment is needed to make PEIs protonated. $R$ remains almost the same between $\mathrm{pH}$ 4.5 and 8.0. This is due to, in limited space, most amine groups have already ionized when $\mathrm{pH}$ is lower than 8 . So the decrease of $\mathrm{pH}$ makes almost no difference until it reaches $\mathrm{pH} 4$. When the $\mathrm{pH}$ value is smaller than $4, R$ increases greatly. This is due to, at this point, high concentration of $\mathrm{H}^{+}$forces the amine groups, which are covered in the multilayers, to be protonated and increases the amount of positive charges. Two sudden changes can be observed from Figure 6, which indicates two $\mathrm{p} K_{\mathrm{a}}$. They are about 8.2 and 3.5, respectively.

The Current Response Dependence on Radius. The influence of tip radius with two different structures of PEIs on $R$ has also been investigated. Figure 7 also shows maxima for the rectification factor due to the interplay of the DDL and the polarization effect at the tip of nanopipette. One possible reason is that a voltage drop may be formed at the tip region because of the access resistance and polarization effects. Thus, the ICR turns to be weaker. Besides, the decrease of 

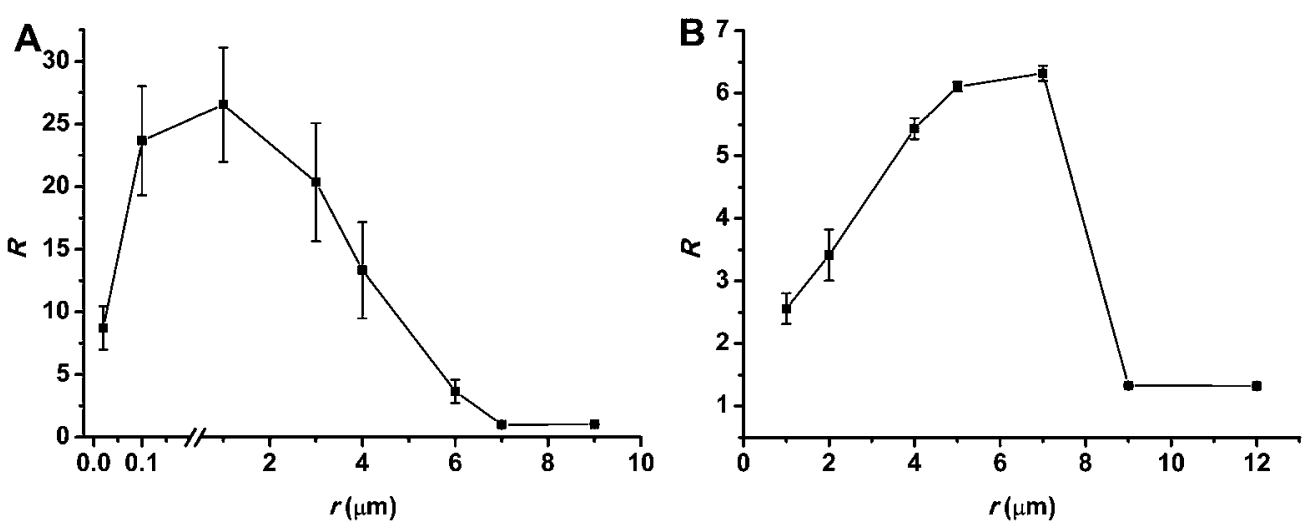

Figure 7. Dependence of ion current rectification on tip radius of nanopipettes for PEIs-coated pipettes. (A) Linear PEIs-coated pipettes, $R=i_{+} / i_{-}$. (B) Branched PEIs-coated pipettes. The $R$ is smaller because it is calculated by current values at $\pm 0.5 \mathrm{~V}$ rather than $\pm 1 \mathrm{~V}$.

rectification at smaller tip diameters is probably related to the fact that the half-cone angle of the pipet is changing, causing the loss of rectification (straight channels, like tubes, normally do not show rectifying properties, but the maximum rectification in conical pores are normally observed at small angles $2-10$ degrees). ${ }^{18,45}$ On the other hand, the tip may lack appreciable ionic selectivity when the tip opening is much larger than the DDL, which also leads to a smaller $R$ value. ${ }^{46,47}$

When linear structure is used, $R$ reaches a peak value with the radius is nearly $1 \mu \mathrm{m}$. However, the peak value shifts to $6 \mu \mathrm{m}$ when branched PEIs are coated. The $R$ is smaller because it is calculated by current values at $\pm 0.5 \mathrm{~V}$ rather than $\pm 1 \mathrm{~V}$ in Figure 7B. But the relationship between $R$ and tip radius is not altered. According to the calculation mentioned above, the $\mathrm{DDL}$ is $10 \mathrm{~nm}$ when the concentration of $\mathrm{KCl}$ is $1 \mathrm{mM}$, and the effective radius of pipettes coated with linear PEIs is $10 \mathrm{~nm}$ when the $R$ reaches its peak value. The effective radius is reduced by 600 times when branched PEIs are used, which is more efficient compared with the results when linear structure is used. This is because more positive charges could be gathered on the inner wall when branched structures are employed. Using a modifier with more positive charges, we may reduce the radius more effectively. ${ }^{48}$ Applications of nanochannels rely on the unique ion transport properties of very small diameter pores. Using this method such applications can be realized in larger channels, e.g., $1 \mu \mathrm{m}$ or larger, which are easier to manipulate for laboratories with limited nanofabrication facilities. Moreover, the performance of subnano sized channels can also be investigated using fabricated nanochannels. Of course, the problem with this sticky polyelectrolyte is that the pipet will have more chances to be blocked and the successful rate is low.

\section{CONCLUSIONS}

In conclusion, this work has presented a relatively simple method of glass pipettes modification with PEIs with high success rate. The smallest radius can be modified by this way is about $3 \mathrm{~nm}$. The surface charge of nanopipettes coated with PEIs can be inverted, and the charge density can also be greatly increased. The sizes of the PEIs have been evaluated by dynamic light scattering and to be 4.1 and $5.3 \mathrm{~nm}$ for linear and branched PEIs, respectively. The effect of sizes of PEIs for the modification is also discussed.

The influences of $\mathrm{KCl}$ concentration, solution $\mathrm{pH}$, and tip radius on the ICR have been investigated in detail. In a much wider range of glass pipettes with radii from several nano- to few micrometers, and rather wider range of $\mathrm{KCl}$ concentration (from $0.01 \mathrm{mM}$ to $100 \mathrm{mM}$ ), the ion current rectification phenomena have been observed. A peak-shape relationship of $R$ on $\mathrm{KCl}$ concentration and radii of pipettes are obtained. Compared with bare glass pipettes, the tip orifice for ICR to happen can be extended to nearly $1 \mu \mathrm{m}$ after modification which means the effective radius can be reduced about 2 orders of magnitudes. Indeed, the exact reason for the case of a DDL bigger than the radius needs to be further explored.

From the relationship between $R$ and $\mathrm{pH}$, two values of $\mathrm{p} K_{\mathrm{a}}$ of PEIs on the surface are estimated to be 8.2 and 3.5. This actually provides a novel way to evaluate the $\mathrm{p} K_{\mathrm{a}}$ of polyelectrolyte on glass walls.

\section{ASSOCIATED CONTENT}

\section{S Supporting Information}

Text and Figure S1. This material is available free of charge via the Internet at http://pubs.acs.org.

\section{AUTHOR INFORMATION}

\section{Corresponding Author}

*Phone: +86-10-62759394. Fax: +86-10-62751708. E-mail: yhshao@pku.edu.cn.

Notes

The authors declare no competing financial interest.

\section{ACKNOWLEDGMENTS}

The financial support for this work from the National Natural Science Foundation of China (20735001 and 21075004), the Innovation Team Programs of the Ministry of Education of China, and the Swiss National Foundation Grant 20PA21 121570/1 are gratefully acknowledged.

\section{REFERENCES}

(1) Gorelik, J.; Gu, Y.; Spohr, H. A.; Shevchuk, A. I.; Lab, M. J.; Harding, S. E.; Edwards, C. R. W.; Whitaker, M.; Moss, G. W. J.; Benton, D. C. H.; Sánchez, D.; Darszon, A.; Vodyanoy, I.; Klenerman, D.; Korchev, Y. E. Biophys. J. 2002, 83, 3296-3303.

(2) Li, Q.; Xie, S.; Liang, Z.; Meng, X.; Liu, S.; Girault, H. H.; Shao, Y. Angew. Chem., Int. Ed. 2009, 48, 8010-8013.

(3) Takahashi, Y.; Shevchuk, A. I.; Novak, P.; Murakami, Y.; Shiku, H.; Korchev, Y. E.; Matsue, T. J. Am. Chem. Soc. 2010, 132, 1011810126. 
(4) Elsamadisi, P.; Wang, Y.; Velmurugan, J.; Mirkin, M. V. Anal. Chem. 2011, 83, 671-673.

(5) Hille, B. Ionic Channels of Excitable Membranes, 2 ed.; Sinauer Associates, Inc.: Sunderland, MA, USA, 1992.

(6) Oliver, D.; Baukrowitz, T.; Fakler, B. Eur. J. Biochem. 2000, 267, 5824-5829.

(7) Wei, C.; Bard, A. J.; Feldberg, S. W. Anal. Chem. 1997, 69, 46274633.

(8) Siwy, Z. S. Adv. Funct. Mater. 2006, 16, 735-746.

(9) Umehara, S.; Pourmand, N.; Webb, C. D.; Davis, R. W.; Yasuda, K.; Karhanek, M. Nano Lett. 2006, 6, 2486-2492.

(10) Karnik, R.; Duan, C.; Castelino, K.; Daiguji, H.; Majumdar, A. Nano Lett. 2007, 7, 547-551.

(11) Ali, M.; Yameen, B.; Cervera, J.; Ramirez, P.; Neumann, R.; Ensinger, W.; Knoll, W.; Azzaroni, O. J. Am. Chem. Soc. 2010, 132, 8338-8348.

(12) Siwy, Z.; Heins, E.; Harrell, C. C.; Kohli, P.; Martin, C. R. J. Am. Chem. Soc. 2004, 126, 10850-10851.

(13) Kovarik, M. L.; Zhou, K.; Jacobson, S. C. J. Phys. Chem. B 2009, 113, 15960-15966.

(14) Yusko, E. C.; An, R.; Mayer, M. ACS Nano 2010, 4, 477-487.

(15) Ramirez, P.; Apel, P. Y.; Cervera, J.; Mafe, S. Nanotechnology 2008, 19, 315707/1-315707/12.

(16) Guerrette, J. P.; Zhang, B. J. Am. Chem. Soc. 2010, 132, 1708817091.

(17) Momotenko, D.; Girault, H. H. J. Am. Chem. Soc. 2011, 133, 14496-14499.

(18) Momotenko, D.; Cortés-Salazar, F.; Josserand, J.; Liu, S.; Shao, Y.; Girault, H. H. Phys. Chem. Chem. Phys. 2011, 13, 5430-5440.

(19) Woermann, D. Nucl. Instrum. Methods Phys. Res., Sect. B 2002, $194,458-462$.

(20) Woermann, D. Phys. Chem. Chem. Phys. 2003, 5, 1853-1858.

(21) Cervera, J.; Schiedt, B.; Ramirez, P. Europhys. Lett. 2005, 71, $35-41$.

(22) White, H. S.; Bund, A. Langmuir 2008, 24, 2212-2218.

(23) Fu, Y.; Tokuhisa, H.; Baker, L. A. Chem. Commun. 2009, 45, 4877-4879.

(24) Vlassiouk, I.; Kozel, T. R.; Siwy, Z. S. J. Am. Chem. Soc. 2009, 131, 8211-8220.

(25) Sa, N.; Fu, Y.; Baker, L. A. Anal. Chem. 2010, 82, 9963-9966.

(26) Kalman, E. B.; Vlassiouk, I.; Siwy, Z. S. Adv. Mater. 2008, 20, 293-297.

(27) Vilozny, B.; Actis, P.; Seger, R. A.; Vallmajo-Martin, Q.; Pourmand, N. Anal. Chem. 2011, 83, 6121-6126.

(28) Actis, P.; Vilozny, B.; Seger, R. A.; Li, X.; Jejelowo, O.; Rinaudo, M.; Pourmand, N. Langmiur 2011, 27, 6528-6533.

(29) Amara, M.; Kerdjoudj, H. Talanta 2003, 60, 991-1001.

(30) Chen, J.; Zeng, F.; Wu, S.; Su, J.; Zhao, J.; Tong, Z. Nanotechnology 2009, 20, 365502.

(31) Xia, T.; Kovochich, M.; Liong, M.; Meng, H.; Kabehie, S.; George, S.; Zink, J. I.; Nel, A. E. ACS Nano 2009, 3, 3273-3286.

(32) Xu, Z.; Shen, G.; Xia, X.; Zhao, X.; Zhang, P.; Wu, H.; Guo, Q.; Qian, Z.; Wei, Y.; Liang, S. J. Transl. Med. 2011, 9, 46-55.

(33) Cui, R. F.; Li, Q.; Gross, D. E.; Meng, X.; Li, B.; Marquez, M.; Yang, R.; Sessler, J. L.; Shao, Y. H. J. Am. Chem. Soc. 2008, 130, 14364-14365.

(34) Beattie, P. D.; Delay, A.; Girault, H. H. J. Electroanal. Chem. 1995, 380, 167-175.

(35) Liu, S. J.; Li, Q.; Shao, Y. H. Chem. Soc. Rev. 2011, 40, 22362253.

(36) Ji, T.; Liang, Z.; Zhu, X.; Wang, L.; Liu, S.; Shao, Y. Chem. Sci. 2011, 2, 1523-1529.

(37) Shao, Y.; Mirkin, M. V. J. Am. Chem. Soc. 1997, 119, 81038104.

(38) Hu, H.; Xie, S.; Meng, X.; Jing, P.; Zhang, M.; Shen, L.; Zhu, Z.; Li, M.; Zhuang, Q.; Shao, Y. Anal. Chem. 2006, 78, 7034-7039.

(39) Parks, G. A. Chem. Rev. 1965, 65, 177-198.

(40) Senn, B. C.; Pigram, P. J.; Liesegang, J. Surf. Interface Anal. 1999, $27,835-839$.
(41) Laforge, F. O.; Carpino, J.; Rotenberg, S. A.; Mirkin, M. V. Proc. Natl. Acad. Sci. U. S. A. 2007, 104, 11895-11900.

(42) Wang, Y. X.; Velmurugan, J.; Mirkin, M. V.; Rodgers, P. J.; Kim, J.; Amemiya, S. Anal. Chem. 2010, 82, 77-83.

(43) Bard, A. J.; Faulkner, L. R. Electrochemical Methods: Fundamentals and Applications; John Wiley \& Sons: New York, 1980.

(44) Sun, J.; Paik, H.; Hwang, B. K. Bioorg. Chem. 1994, 22, 318327.

(45) Perry, J. M.; Zhou, K.; Harms, Z. R.; Jackson, S. C. ACS Nano 2010, 4, 3897-3902.

(46) Vlassiouk, I.; Smirnov, S.; Siwy, Z. ACS Nano 2008, 2, 15891602.

(47) Vlassiouk, I.; Smirnov, S.; Siwy, Z. Nano Lett. 2008, 8, 19781985.

(48) Tagliazucchi, M.; Rabin, Y.; Szleifer, I. J. Am. Chem. Soc. 2011, 133, 17753-17763. 